\title{
ERROS POTENCIAIS NA ESTIMATIVA DA MINERALIZAÇÃO DO NITROGÉNIO DO SOLO
}

\section{POTENTIAL ERRORS IN ESTIMATING SOIL NITROGEN MINERALIZATION}

\author{
FIávio Anastácio de Oliveira Camargo ${ }^{1}$ Clesio Gianello $^{1}$ Caio Vidor $^{1}$
}

\section{-NOTA-}

RESUMO

Avaliou-se a ocorrência de N-orgânico solúvel em experimentos para estimativa da mineralização do nitrogénio de dez solos do Rio Grande do Sul. Foi utilizado o procedimento incubação-lixrviação e extração com $\mathrm{CaCI}_{2} 0,01 \mathrm{~mol} \mathrm{~L}^{-1}$, onde foi determinado a concentração de $\mathrm{N}$-mineral, $\mathrm{N}$-total e $\mathrm{N}$-orgânico solúvel no extraio. Constatou-se que a lixiviação periódica do solo retira porção considerável de $N$-orgânico solúvel e assim subestimando a estimativa da mineralização do nitrogénio do solo. Palavras-chave: $\begin{aligned} & \text { N-mineralizado, incubação-lixiviação, in- } \\ & \text { orgânico solúvel. }\end{aligned}$

SUMMARY

The occurrence of organic $N$ was delermined $m$ experiments on $N$ mineralization in ten soilsfrom Rio Grande do Sul State, Brazil. The incubation-leaching method, with $\mathrm{CaCI}_{2}$ $0,01 \mathrm{~mol} \mathrm{~L}^{-1}$, was used and the leveis of mineral, total and soluble organic nitrogen in the extract measured. Periodic leaching of the soil removed a considerable portion ofthe soluble organic $N$ and thus underestimating the nitrogen mineralization in the soil.

Key words: Mineralized-N, incubation-leaching, soluble organic
A maioria dos estudos envolvendo a avaliação da disponibilidade do nitrogénio $(\mathrm{N})$ do solo tem utilizado índices baseados em métodos químicos e biológicos. O método biológico de incubação aeróbica em laboratório (STANFORD \& SMITH, 1972), provavelmente é o mais adequado para aproximações com as respostas a campo. Neste método, a mineralização periódica do nitrogénio é obtida através da incubação do solo em tubos para lixiviação. Uma limitação considerável que ocorre neste procedimento é a remoção de nitrogénio orgânico solúvel durante o processo da lixiviação. Esta forma solúvel facilmente mineralizável não é contabilizada no processo da mineralização, gerando assim, estimativas erróneas do nitrogénio potencialmente mineralizável (CAMARGO, 1996). A avaliação da ocorrência desta forma orgânica solúvel foi o objetivo do presente trabalho.

Os solos utilizados neste experimento foram coletados do horizonte superficial $(0-20 \mathrm{~cm})$ de dez solos representativos do Estado do Rio Grande do Sul, compreendendo uma ampla faixa de teores de matéria orgânica (M.O.) (17 a $\left.56 \mathrm{~g} \mathrm{~kg}^{-1}\right), \mathrm{N}\left(0,9\right.$ a 3,6 $\left.\mathrm{g} \mathrm{kg}^{-1}\right)$, $\mathrm{pH}(4,0$ a 6,4$)$ e conteúdo de argila (180 a $\left.550 \mathrm{~g} \mathrm{dm}^{-3}\right)$.

${ }^{1}$ Professor do Departamento de Solos, Faculdade de Agronomia, Universidade Federal do Rio Grande do Sul, Caixa Postal 776, 90001970, Porto Alegre - RS. 
Retirou-se amostras para a incubação em tubos de lixiviação para a determinação do Nmineralizado em função do tempo $(0,2,4,8,16$ e 32 semanas), conforme metodologia descrita por STANFORD \& SMITH (1972), utilizando-se, entretanto, $25 \mathrm{~g}$ de solo por amostra. A lixiviação foi feita com $\mathrm{CaCI}_{2}$ 0,01M, seguida de uma solução nutritiva sem $\mathrm{N}$, aplicando-se sucção uniforme e mantendo-se a umidade e as condições de incubação de acordo com STANFORD \& SMITH (1972). No lixiviado, foi analisado o conteúdo de $\mathrm{N}$-mineral (destilação com $\mathrm{MgO}$ e liga de devarda), o N-total (micro kjeldahl) e o $\mathrm{N}$-orgânico solúvel em $\mathrm{CaCI}_{2}$ (N-total - N-mineral).

A presença de N-orgânico solúvel foi constatada já no tempo zero de incubação, sendo este valor, cerca de $47 \%$ do N-total lixiviado neste período (Tabela 1). Em muitos casos, o N-orgânico inicial é frequentemente maior que o $\mathrm{N}$-mineral. Esta situação reflete a presença de materiais orgânicos prontamente decomponíveis e de compostos orgânicos nitrogenados, degradados ou liberados para o meio em função do preparo dado às amostras antes da incubação. Entretanto, os maiores valores para $\mathrm{N}$-total, $\mathrm{N}$-mineral e $\mathrm{N}$-orgânico foram obtidos na segunda semana de lixiviação, ao contrário dos resultados apresentados por SMITH (1987), que verificou este comportamento no tempo inicial de incubação. Aparentemente, diferenças podem ter ocorrido em função de que os solos do presente trabalho permaneceram em baldes com condições de atingir o equilíbrio natural, por mais de três anos antes da realização do experimento, o que conduziria a um menor erro decorrente do manejo da amostra.

Constatou-se em termos de valores médios dos dez solos analisados que o N-orgânico solúvel representou $36,6 \%$ do $\mathrm{N}$-total acumulado nas

Tabela 1 - Valores acumulados ${ }^{1}$ de $\mathrm{N}$-total, N-mineral e N-orgânico (mg $\mathrm{kg}^{-1}$ ) nos lixiviados da $\mathrm{CaCl}_{2} 0,01 \mathrm{~mol} \mathrm{~L}^{-1}$ em diferentes tempos de inoculação.

\begin{tabular}{ccccccc}
\hline & \multicolumn{5}{c}{ Tempo (semanas) } \\
\cline { 2 - 7 } & 0 & 2 & 4 & 8 & 16 & 32 \\
\hline N-total & 21,8 & 16,5 & 154,8 & 194,9 & 218,2 & 240,3 \\
N-mineral & 11,5 & 67,5 & 98,4 & 127,1 & 140,1 & 152,2 \\
N-orgânico & 10,3 & 39,2 & 56,4 & 67,8 & 78,1 & 88,1 \\
\hline
\end{tabular}

${ }^{1}$ Médias de 10 solos do Rio Grande do Sul.
32 semanas. Esta percentagem foi inferior à obtida por SMITH et al., (1980), em cerca de 12,4\% em solos incubados durante 11 semanas, que em termos totais foi obtido em média para as 11 semanas, $50,63 \mathrm{mg} \mathrm{kg}^{-1}$ de N-orgânico solúvel. No presente trabalho, este valor foi verificado na quarta semana, atingindo um máximo de $88,1 \mathrm{mg} \mathrm{kg}^{-1}$ ao fmal da $32^{\mathrm{a}}$ semana. Em termos de distribuição, constatou-se que com o aumento do conteúdo de M.O. (r=-0,818; $\mathrm{p}<0,001)$ e $\mathrm{N}(\mathrm{r}=-0,775 ; \mathrm{p}<0,001)$ ocorreu uma diminuição na concentração de N-orgânico solúvel. Provavelmente, os solos com maior conteúdo de M.O. apresentam-se com uma maior estabilidade e recalcitrância da molécula húmica, diminuindo desta forma, a sua concentração em solução.

A partir destes resultados, constata-se nitidamente que a lixiviação periódica do solo com solução salina, em experimentos de incubação para avaliar o N-mineralizado, também retira do sistema grande quantidade de N-orgânico solúvel, provavelmente, de fácil mineralização, subestimando, assim, a mineralização de $\mathrm{N}$ nos períodos subsequentes de incubação. É necessário um fator de correção para os dados de $\mathrm{N}$ mineralizado do o N-total contido no lixiviado. Apartir das condições experimentais e considerando o tempo zero, este fator foi de $\mathrm{N}$-total $=\mathrm{N}$ mineralizado. $1,73 \pm 0,18$, enquanto que a partir da segunda semana este fator $(1,56)$ apresentou-se mais uniforme, com um baixo desvio padrão da média $( \pm$ 0,034). Todavia, esta comparação somente deverá ser utilizada se não estiver ocorrendo mineralização do N-orgânico solúvel, situação esta que ainda não foi testada e comprovada na literatura (CAMARGO, 1996).

\section{REFERENCIAS BIBLIOGRÁFICAS}

CAMARGO, F.A. de O. F racionamento e dinâmica do nitrogénio orgânico em solos do Rio Grande do Sul. Porto Alegre -RS. 152 p. Tese (Doutorado em Ciência do Solo) - Curso de pós-graduaçâo em Agronomia Universidade Federal do Rio Grande do Sul, 1996.

SMITH, S.J. Soluble organic nitrogen losses associated with recovery of mineralized nitrogen. Soil Science Society of AmericanJournal,Madison,v.51,p. 11911194, 1987.

SMITH, J.L., SCHNABEL, B.L., McNEAL, B.L., et al Potential errors in the first-order model for estimating soil nitrogen mineralization potentials. Soil Science Society of American Journal, Madison, v. 44, p. 9961000, 1980.

STANFORD, G., SMITH, S.J. Nitrogen mineralization potentials of soils. Soil Science Society of American Proceedings, adison, v. 36, p. 465-472, 1972. 